\title{
NOTE ON A CLASS OF HARMONIC FUNCTIONS
}

BY G. C. EVANS

1. Introduction. Let $S$ be a multiply-connected open region, bounded by an exterior circle $s_{0}$ and $n$ interior circles $s_{1}, \cdots, s_{n}$, not touching, and let $u(P)$ be harmonic, not necessarily bounded, in $S$. The author has shown that a necessary and sufficient condition that $u(P)$ be given as the sum of logarithmic terms and Poisson-Stieltjes integrals around the several boundaries is that the integrals $\int_{0}^{2 \pi}|u| d \theta_{i}$, extended around circles concentric with each boundary circle, remain bounded in the neighborhood of each boundary circle. He has shown also that a necessary and sufficient condition that $u(P)$ be given by the corresponding Stieltjes integral generalization of the formula in terms of the normal derivative of the Green's function is that $\int|u| d h$ remain bounded, where the integration is extended over the whole of a variable curve $g=$ const., with $g$ the Green's function and $h$ its conjugate, referred to some fixed pole $Q$. Finally the author has shown that a necessary and. sufficient condition for the latter formula is that $u(P)$ be the difference of two functions harmonic and not-negative in $S .^{*}$ It is desired to show that this condition is also necessary and sufficient for the former mode of representation, so that the two forms of representation apply to the same class of functions. That the condition is necessary follows at once from the mode of representation. It is only the sufficiency that requires proof.

2. Lemma I. Let $S_{0}$ be a simply-connected open region, bounded internally or externally by a circle $s_{0}$, of radius

* The Dirichlet problem for the general open finitely-connected region, Proceedings of the International Congress at Toronto (1924). 
$R_{0}$, and let $u(P)$ be the difference of two not-negative harmonic functions $u^{\prime}(P), u^{\prime \prime}(P)$ in an annular neighborhood in $S_{0}$ of the boundary $s_{0}$. Then $\int_{0}^{2 \pi}|u| d \theta$ is bounded for the circles of that neighborhood concentric with $s_{0}$.

For convenience let $S_{0}$ be an interior region. Let $s^{\prime}$, of radius $r^{\prime}$, be a fixed circle in the annular neighborhood and let $s$ be a circle of radius $r, r^{\prime} \leqq r<R_{0}$, all these circles having the same center 0 . The lemma follows immediately from Bôcher's device, evaluating the surface integral of $(1 / r) \partial u / \partial r$. In fact, by integrating this expression in $u^{\prime}$ over the annular region between $s^{\prime}$ and $s$, and equating its two evaluations as an iterated integral, we have the equation

$$
\left(\int_{0}^{2 \pi} u^{\prime} d \partial\right)_{r}=\left(\int_{0}^{2 \pi} u^{\prime} d \theta\right)_{r^{\prime}}+\alpha_{1} \log r-\alpha_{1} \log r^{\prime}
$$

where $\alpha_{1}$ is a constant. Hence

$$
\int u^{\prime} d \theta=\int\left|u^{\prime}\right| d \theta
$$

is bounded. Similarly $\int\left|u^{\prime \prime}\right| d \theta$ is bounded, and therefore $\int|u| d \theta$ is bounded.

3. Lemma II. In the open region $S$ of $\S 1$, let $u(P)$ be the difference of two functions $u^{\prime}(P), u^{\prime \prime}(P)$, harmonic and not-negative in $S$. Then $u(P)$ is the sum of $n+1$ functions $u_{i}(P)$, each harmonic except for possible logarithmic singularities at infinity, in the simply-connected open region $S_{i}$ bounded by $s_{i}$, and each satisfying in its region $S_{i}$ the conditions of Lemma 1.

The first part of this lemma is proved by Osgood.* The last part follows immediately by writing, in the neighborhood of $s_{i}, u_{i}(P)$ as the difference $u(P)-\bar{u}(P)$ where

$$
\bar{u}(P)=\sum_{j}^{\prime} u_{j}(P)
$$

the summation not including $j=i$.

* Osgood, Lehrbuch der Funktionentheorie, Leipzig and Berlin, 1912, pp. $642-644$. 
4. Proof of the Theorem. The statement $\S 1$ can now be proved. In fact, if the hypothesis of Lemma 2 holds, $\int\left|u_{i}\right| d \theta_{i}$ is bounded for circles concentric with $s_{i}$ in the annular neighborhood in $S$ of $s_{i}$, by Lemma 1 . The same is true for $\int\left|u_{j}\right| d \theta_{i}, j \neq i$, since $u_{j}(P)$ is harmonic on $s_{i}$ itself if $j$ is unequal to $i$. But

$$
\int|u| d \theta_{i} \leqq \sum_{j=0}^{n} \int\left|u_{j}\right| d \theta_{i} .
$$

Hence the condition of the first cited theorem of $\S 1$ is satisfied. This is what was to be proved.

4. Conclusion. If we make a conformal transformation of $S$ into a general open region $T$ of the same connectivity, without isolated point boundaries, the Toronto memoir already cited shows that the condition that $u(P)$ be the difference of two not-negative harmonic functions is necessary and sufficient that $u(P)$ be given by a generalized Stieltjes integral in terms of the conjugate to the Green's function for $T$ on the ordered boundary points of $T$. But if we make conformal transformations of each $S_{i}$ into the corresponding simply-connected region $T$, we obtain a representation of $u$ as the sum of terms $u_{i}$ where each $u_{i}$ is the sum of a possible logarithmic term and a generalized Stieltjes integral on the frontier points of $T_{i}$, expressed in terms of the conjugate to the Green's function for $T_{i}$. And from what we have proved in $\S 3$, it follows that this representation applies to precisely the same class of harmonic functions as the other. The Dirichlet problem is a special case of the problem here discussed.

The connection between the two forms of representation of $u(P)$ is given by integral equations which are worth study on their own account.

The Rice Institute 